\title{
Label-Free Plasmonic Detection of Untethered Nanometer-Sized Brownian Particles
}

\author{
Martin Dieter Baaske, Peter Sebastian Neu, and Michel Orrit* \\ Cite This: ACS Nano 2020, 14, 14212-14218 \\ Read Online
}

ACCESS | Lلll Metrics \& More | 国 Article Recommendations ｜ sl Supporting Information

ABSTRACT: Optical detection of individual nanometer-sized analytes, virus particles, and protein molecules holds great promise for understanding and control of biological samples and healthcare applications. As fluorescent labels impose restrictions on detection bandwidth and require lengthy and invasive processes, label-free optical techniques are highly desirable. Here, we introduce an optical technique capable of transforming gold nanorods commonly used as photostable labels into highly localized high-speed probes. Our method detects

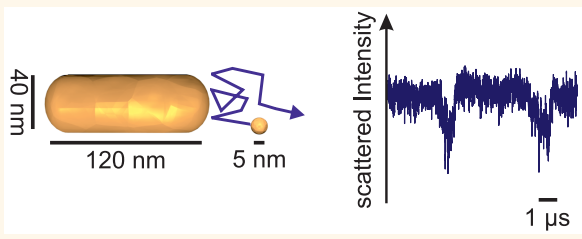
single untethered $5 \mathrm{~nm}$ diameter gold particles as they traverse subattoliter volumes in Brownian motion with a time resolution below microseconds.

KEYWORDS: label-free, scattering, near field, LSPR, micelles, nanoparticles, microemulsion

$\mathrm{P}$ owerful label-free optical methods have recently emerged, such as interferometric scattering microscopy, ${ }^{1-5}$ plasmonic nanoparticle-based assays, ${ }^{6-10}$ and microcavity-based assays. ${ }^{11-21}$ Although highly sensitive, these methods are often restricted to integration times in excess of microseconds. This often imposes a requirement to impede analyte motion during these periods via specific molecular tethers, unspecific adsorption, or confining arrangements. ${ }^{22-25}$ Label-free optical techniques so far regularly rely on chemical tethers or other means that impede the analyte's motion. These chemical tethers or receptor molecules fulfill a dual purpose. First they provide the specificity; i.e., they ideally only interact with one species of target molecules and thereby provide selective identification. Their second purpose is to hold the target analyte fast for time periods long enough to enable detection. The requirement for chemical specificity can be relaxed to a large extent if the assay directly or indirectly measures several physical properties of the analyte, i.e., its charge, mass, or polarizability. Then, unspecific adsorption to a surface is sufficient. ${ }^{5}$ For plasmonic nanoparticle-based assays, permanent or long adsorption duration is undesirable as their limited surface area allows only a few analytes to bind. Highly specific chemical interaction, however, usually entails high affinity (i.e., strong bonds), making the multiplexed read out of many particles a necessity. ${ }^{8,9}$ Consequently, a nanoparticlebased method which would lift the requirement for specific chemical modifications would be highly advantageous but requires accurate determination of more physical properties, such as Stokes radius or charge, to enable identification. However, to provide access to these properties, such a sensor must probe subattoliter volumes and therefore needs to be fast. The current state of the art for on-the-fly plasmonic detection was established by Wulf $e$ t al., who detected diffusing particles as they propagate through a sensing nanorod's near field. ${ }^{26}$ Their method required tracking the whole spectrum of the rod's plasmon resonance and therefore was limited to $\sim 0.1 \mathrm{~ms}$ time resolution and comparatively large particles (diameter $\geq 20 \mathrm{~nm}$ ). In the following, we will demonstrate an optical method that improves the time resolution $10^{4}$-fold and the sensitivity with respect to analyte polarizability more than 100 fold and as a consequence is capable of recognizing even single analytes.

\section{RESULTS AND DISCUSSION}

In order to resolve such short-lived and minute intensity perturbations due to shifts of a gold nanorod's (GNR) longitudinal surface plasmon resonance (LSPR, frequency $\nu_{0}$, half width at half-maximum $\Gamma$ ), one must overcome fundamental noise sources (essentially photon shot noise) as well as experimental noise from laser, detector, and residual vibrations and drifts of the setup. ${ }^{27}$ To do so, one must optimize the signal-to-noise ratio for fluctuations in detected power caused by shifts of the GNR's plasmon resonance.

We do this via the confocal microscopy setup shown in Figure 1A, which allows us to utilize the intrinsic scattering anisotropy of GNRs. As depicted in Figure 1B this in principle

Received: August 31, 2020

Accepted: October 7, 2020

Published: October 15, 2020 
A

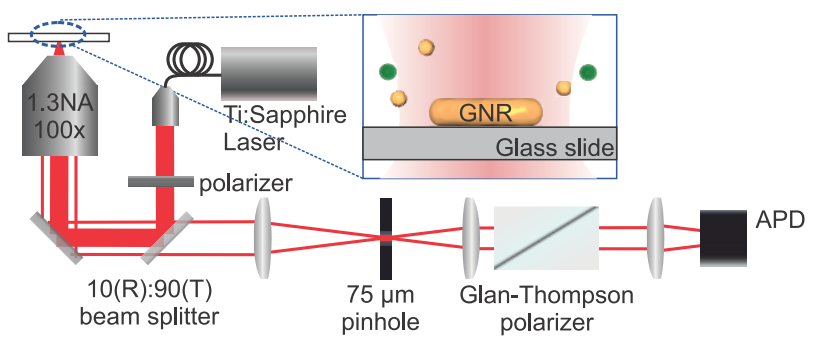

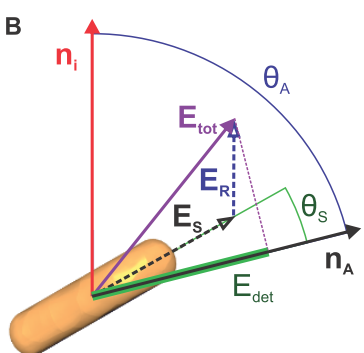

Figure 1. Experimental method: (A) setup used to monitor minute fluctuations of scattered intensity due to perturbations of a GNR's near field (inset) by freely diffusing nanoscopic analytes; $(B)$ ratio of scattered $E_{S}$ to reflected field $E_{\mathrm{r}}$ components contributing to $E_{\mathrm{det}}$ can be chosen by varying the incident field polarization $n_{\mathrm{i}}$ and the analyzer (Glan-Thompson) $n_{\mathrm{A}}$ orientation, i.e., the angles $\theta_{\mathrm{A}}$ and $\theta_{\mathrm{S}}$.

allows us to choose the ratio of reflected $E_{\mathrm{R}}$ over scattered field $E_{S}$ contributions to the detected field component $E_{\text {det }}$ for an arbitrary GNR orientation. The detected power can be expressed as

$$
P_{\operatorname{det}} \propto\left(|\hat{r}|^{2}+|\hat{s}|^{2}+2|\hat{r}||\hat{s}| \cos \beta\right) E_{0}^{2}
$$

where $\hat{r}$ denotes the effective reflection coefficient and is a function of the analyzer angle, $\hat{s}$ denotes the effective scattering coefficient and is a function of the difference $\Delta \nu$ between excitation and LSPR frequency as well as the NR's angle with respect to the polarizer and analyzer angles, and $\beta$ denotes the phase difference between reflected and scattered field and is a function of $\Delta \nu$ and the Gouy phase $\gamma$ (i.e., the NR's and the reflective interface's position in the microscope objective's focus). As a consequence, the signal-to-noise ratio can be optimized for individual NRs by adjusting polarization angles $\Delta \nu$ and the Gouy phase. We express the influence of all these parameters via the dimensionless Tuning function $T$ (see the Supporting Information, section 1), which is proportional to the signal-to-noise ratio. The maximum signal-to-noise can be achieved by making use of the interference term in eq 1 and thus a bright-field scattering configuration, i.e., $|\hat{r}|>|\hat{s}|$ or $|\hat{r}| \approx|\hat{s}|$, which yields a $T_{\max , \mathrm{BF}} \approx 2$. In order to avoid contributions from linearly birefringent components, such as dielectric mirrors and beam splitters, to the analyzed signals we, however, choose to restrict our proof-of-principle measurements to close to crosspolarized configurations. Most of the light scattered by analyte particles will be polarized parallel to the incident field's polarization and will be filtered by the analyzer. As a consequence, the changes in scattered intensity can be unequivocally attributed to changes in the nanorods scattering cross-section, i.e., shifts of its LSPR. This configuration is equivalent to dark-field scattering. The maximum $T$-value that can be achieved in this configuration, i.e., $|\hat{r}| \approx 0$ is $T_{\max , \mathrm{DF}} \approx$ 0.7. Another parameter that has to be considered is the temperature increase of the NRs for which we find $\Delta T \lesssim 8 \mathrm{~K}$ under our experimental conditions (Supporting Information, section S2). Changes in the temperature profile around the NR can cause (a) deviations of the local viscosity, (b) thermophoresis, (c) irreversible damage of analyte (i.e., denaturation), and (d) at high powers reshaping of the nanorod. While some of these effects may even be desirable for future studies, we tried to keep the temperature change as low as possible for our proof-of-principle experiments.

We record continuous $0.1 \mathrm{~ms}$ traces of scattered intensity changes: $\tilde{I}(t)=I(t)-\langle I\rangle$, where $I(t)$ is the intensity at the time $t$ and $\langle I\rangle$ the average intensity. From these we compute the respective normalized autocorrelation curves

$$
G(\tau)=\frac{\langle\tilde{I}(t+\tau) \tilde{I}(t)\rangle}{\left\langle\tilde{I}^{2}\right\rangle}
$$

where $\tau$ denotes the temporal delay from each entire trace using the Wiener-Khinchin theorem in order to obtain ensemble properties. Note that we normalize to the variance of the intensity fluctuations instead of the squared average intensity because we use an AC-coupled detector in order to reject low-frequency noise. Computation of autocorrelations does not involve the single-event detection algorithms as described in Supporting Information, section S6. In addition to autocorrelation curves from single traces, we also discuss averages $G_{N}(\tau)$ over $N$ traces. We find that all analytes discussed in the following exhibit autocorrelation curves that are fitted well with stretched exponentials

$$
G_{\mathrm{fit}}(\tau)=A e^{-\left(\tau / \tau_{D}\right)^{\beta}}
$$

where $A$ denotes the amplitude, $\beta$ the stretch-exponent, and $\tau_{\mathrm{D}}$ the decay time.

Recognition of Fluctuations in Local Nanodroplet Concentrations. In order to show that our sensor can obtain information in a relatively crowded environment, i.e., at analyte concentrations in excess of $0.1 \mathrm{mM}$, which are usually not accessible to image-based methods, we have prepared a microemulsion of oil in water. Microemulsions are stable physical phases of ternary surfactant-oil-water mixtures and do not suffer from the drawbacks of unspecific sticking that are commonly encountered for proteins. This way, we circumvent the need for chemical surface modifications in these pilot experiments. Specifically we have chosen a nonionic microemulsion system consisting of a soybean oil/polyoxyethylene10-oleyl ether (Brij-O10)/water mixture ${ }^{28,29}(4 \% / 16 \% / 80 \%)$ that forms stable and monodisperse micelles, or nanodroplets with $(8.1 \pm 2.6) \mathrm{nm}$ diameter, as determined via dynamic light scattering (see Figure S4). As micelles have a refractive index of $1.48,{ }^{30}$ they mimic proteins of $\sim 250 \mathrm{kDa}$ molecular weight in size, shape, and polarizability. The concentration of these solutions is typically $\sim 1 \mathrm{mM}$, i.e., $20 \%$ volume fraction (oil + detergent). In this high concentration regime the unequivocal recognition of single analytes is not possible as more than one analyte particle will regularly be present in the NR's near field. Nonetheless ensemble properties can be obtained from intensity trace autocorrelations.

The intensity traces shown in Figure 2B exhibit clear perturbations of (sub)microsecond duration caused by these microemulsion nanodroplets-the positive and negative signs of these events and their high rate of occurrence suggest that these cannot be unequivocally contributed by single micelles, but rather stem from number fluctuations of nanodroplets in 

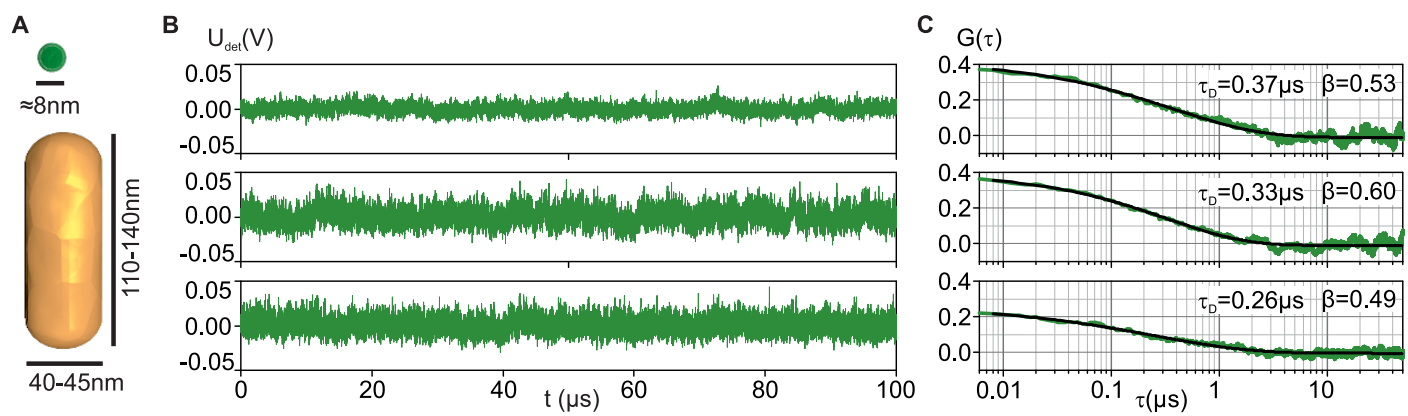

Figure 2. Concentration fluctuations in a microemulsion: (A) dimensions of the sensor rod and the microemulsion nanodroplets simulating $250 \mathrm{kDa}$ proteins; (B) $0.1 \mathrm{~ms}$ long intensity traces which exhibit submicrosecond variations of the scattered intensity caused by number fluctuations of nanodroplets. The three traces were recorded on three different rods.(C) Corresponding autocorrelation curves (green) together with their stretched exponential fits (black).

the near field. Even autocorrelation curves of $0.1 \mathrm{~ms}$ intervals exhibit significant contrast despite submicrosecond relaxation times (see Figure 2C). Averaged autocorrelations $\left(G_{1000}\right)$ obtained from different gold nanorods in different microemulsion samples show only minor deviations with respect to $\beta$. The $\tau_{\mathrm{D}}$ times, however, range from 0.25 to $1.0 \mu \mathrm{s}$ (compare also Figure S4). These differences in $\tau_{\mathrm{D}}$ and $\beta$ might well reflect individual differences in size and shape of the single GNRs and their respective near-field distributions. High-angle annular dark-field scanning transmission electron microscopy (HAADF-STEM) images of our nanorods samples which depict these differences and the size distribution of the NR samples are shown in Figure S12 and S13 of the Supporting Information. We can determine the diffusion length $L=\sqrt{2 D_{s} \tau_{D}}$, where the $D_{s}=D_{0} /(1+2.5 \phi)$ is the hardsphere model based self-diffusion coefficient ${ }^{31}$ as a function of the volume fraction $\phi(=0.2$ for our system $)$ and the diffusion coefficient $D_{0}$ is given by the Stokes-Einstein equation, associated with our $\tau_{\mathrm{D}}$. From this we find $L=(3-10) \mathrm{nm}$. Here, the higher value is comparable to near-field decay length, ${ }^{26}$ whereas the lower value matches the analyte's radius. The latter case might indicate that the nanodroplets can probe point-like defects on the GNR's surface that are associated with high field strength in their proximity. ${ }^{15,17,32}$ In order to clearly distinguish contributions of analyte from NR properties, however, further detailed studies like the correlation of electron tomographic $\mathrm{NR}^{33,34} 3 \mathrm{D}$-maps with the appearance of correlations on the sub-100-ns scale will be necessary. We also find the amplitude of our autocorrelation curves to decrease with nanodroplet concentration as we stepwise dilute the sample to a quarter of its initial concentration $C_{0}$ (see Figure S4B). This reflects the reduction in the number of nanodroplets perturbing the GNR's near field in a given time interval. We want to note here that usually the autocorrelation's contrast cannot be unequivocally attributed to either the magnitude of single analyte perturbations or the rate at which these occur (Supporting Information, section S4).

Recognition of Single Nanoparticles at Nanomolar Concentrations. In order to investigate our sensor's performance in the submicromolar concentration regime we utilize citrate-capped gold nanoparticles (GNPs) with $5 \mathrm{~nm}$ diameter and concentrations $<50 \mathrm{nM}$.

In this regime, we recognize clear spike/burstlike intensity perturbations due to single particles (Figure 3B,C). We have developed an algorithm that recognizes these perturbations (Supporting Information, section S6). In general, we find that these perturbations show the expected sign dependent on which side of a nanorod's LSPR is probed (see Figure 3A). Specifically, this means an intensity decrease (increase) for NRs probed on the short (long) wavelength side of their LSPRs as analytes with positive excess polarizabilities entering the NRs near field will cause the resonance to shift toward longer wavelengths. We further find that the distributions of interevent durations follow poissonian statistics (see Supporting Information, section S7). Note that the respective autocorrelations as computed from the whole intensity traces and stretched-exponential fits for the example traces in Figure 3 are shown in the Supporting Information (Figure S10). In contrast to these autocorrelations, the data extracted from single-particle events provides access to additional dimensions for analysis, i.e., additional means to discern between analytes and their diffusive behavior with respect to experimental conditions. As shown in Figure 3D we find clear correlations between the mean amplitudes and the durations $(T)$ of individual events. Here, the duration is defined as the time during which the amplitude of an individual burst exceeds a threshold value (see Supporting Information, section S6, for details). The mean amplitude of an event is a measure for the average integrated field strength an individual particles sees along its trajectory through the detection volume. The duration $T$ provides a measure for the time a particle spent inside the detection volume without interruption. Specifically, we find that particles which remain inside the NR's detection volume for longer are also more likely to possess trajectories with higher integrated field strength yielding higher mean amplitudes. This reflects that particles traversing the near field for longer are also more likely to penetrate deeper into the NR's near field toward its tips where the near field is the strongest. Trajectories that bring particles close to the NR's tips have to traverse the detection volume at least twice and thus require longer minimum durations as compared to trajectories which just graze the detection volume's outer boundary. We also find a clear correlation between the maximum amplitudes and the variance of individual events (compare Figure 3E). The maximum amplitude provides a measure for the highest field strength a particle sees along its trajectory, i.e., a proxy for how close an individual particle came to the NR's surface and especially its tips. The variance

$$
\sigma^{2}=\frac{1}{T} \sum_{T}(I(t)-\langle I\rangle)^{2}
$$


A

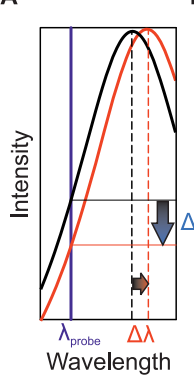

B $U_{\text {det }}(\mathrm{V}$

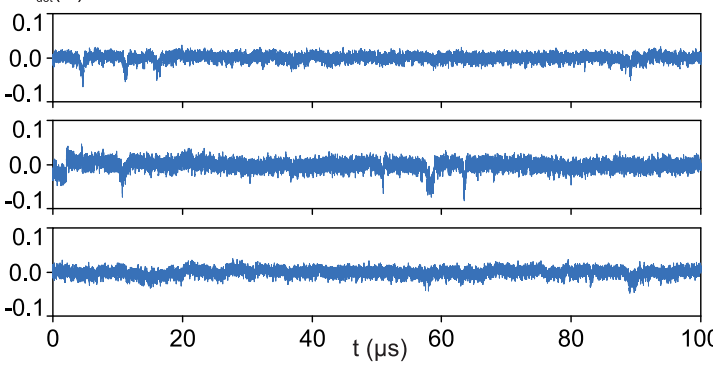

C $U_{\text {det }}(V)$
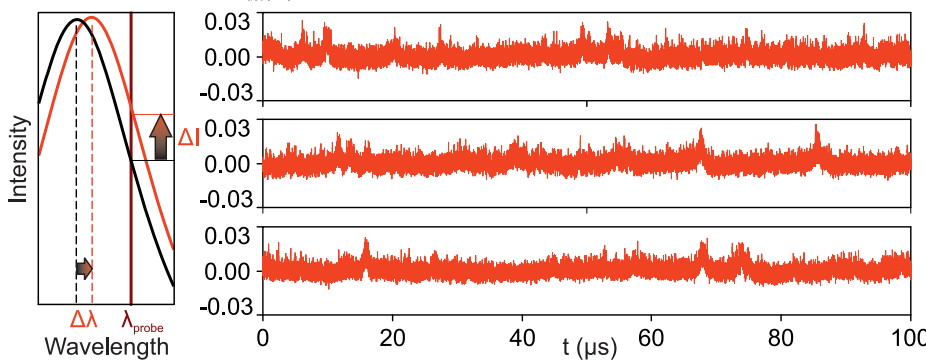
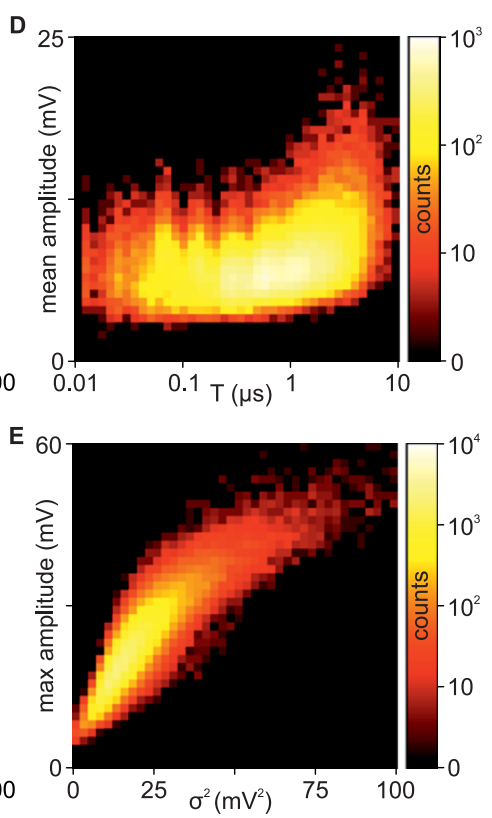

Figure 3. Single-particle detection: (A) conceptual graphics relating the analyte-induced changes of the LSPR's wavelength $\Delta \lambda$ (magnitude exaggerated for visibility) to the respective changes in intensity $\Delta I$ for blue- (top) and red-detuned (bottom) probe wavelength; (B) (bluedetuned $\lambda_{\text {probe }}$ ) and (C) (red-detuned $\lambda_{\text {probe }}$ ) experimental intensity traces showing clear bursts caused by 5 nm diameter gold nanoparticles perturbing the NR's near field; (D, E) distributions of event mean amplitude $v s$ event duration $T$ and maximum amplitude $v s$ variance $\left(\sigma^{2}\right)$, respectively (same NR as C). Both distributions exhibit clear correlations between the corresponding properties.

is a measure for how strong the amplitude $I$ of an individual event fluctuates about its mean $\langle I\rangle$ throughout its duration $T$ and therefore provides a proxy for the overall variation of the field's strength along a particle's trajectory. The NR's near field decays nonlinearly and rapidly with increasing distance from the NR's tips. Thus, we expect that particles with trajectories that enter zones with higher field strength need to diffuse through zones that exhibit stronger field gradients. Indeed, we find this correlation reflected in our data as events with higher maximum amplitudes exhibit higher variance (compare Figure $3 \mathrm{E}$ ). Our simulation results (see Supporting Infomation, section S8) indicate that in future studies this type of analysis can be used to discern between different analytes possibly even on a single-shot basis. This type of discrimination is not directly possible from autocorrelations or via ensemble measurements like traditional dynamic light scattering without prior knowledge of analyte composition. We also want to point out that the single-event analysis can in principle be extended to include higher statistical moments like kurtosis and skewness hence providing additional dimensions for the discrimination of analytes (see Figure S11).

Probing the Effect of lonic Strength on Nanoparticle Detection. The measurements shown in Figure 3 were performed at an ionic strength of $50 \mathrm{mM}$ (3B) and $120 \mathrm{mM}$ (3C, 3D and $3 \mathrm{E})$, respectively. Both the citrate-capped GNP and the nanorods are negatively charged at neutral $\mathrm{pH}$ and thus repel each other. The range of this repulsive interaction can be altered via the solution's ionic strength, i.e., Debyescreening (Supporting Information, section S5). ${ }^{35}$ In fact, we do not recognize any events in the presence of GNPs without additional electrolyte in the solution (Supporting Information, section S6). To further investigate the influence of Debye screening on the diffusion of the $5 \mathrm{~nm}$ diameter GNPs, we record intensity traces starting upon the injection of a small volume $(100 \mu \mathrm{L})$ of a sodium chloride solution at high concentration $(200 \mathrm{mM})$ into the chamber filled with Milli-Q water premixed with $8 \mathrm{nM}$ GNPs $(550 \mu \mathrm{L})$. Following the injection the local ionic strength around the NR will increase over time as the electrolytes diffuse into the chamber until the equilibrium concentration of $30 \mathrm{mM}$ is reached throughout the whole solution. Due to this process, the volume in which the repulsive Coulomb interaction between analyte GNPs and the sensor nanorod can dominate the Brownian motion will decrease over time, allowing the analytes to come ever closer to the NRs surface. This process is reflected in our intensity autocorrelations (Figure 4) as an increase in contrast over time. The shortening of $\tau_{\mathrm{D}}$ values and lowering of stretch exponents $\beta$, however, requires further discussion. The nearfield gradients increase with proximity to the NR's surface. In consequence equal analyte displacements will cause faster and stronger intensity changes for analytes closer to the NR's

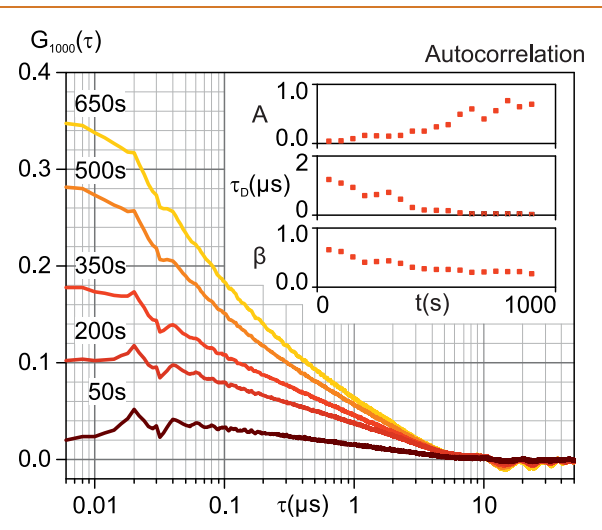

Figure 4. Gold nanoparticle detection at varying ionic strengths: main panel shows the change of averaged trace autocorrelation over time upon injection of sodium chloride (final concentration $30 \mathrm{mM}$ ) alongside the stretched-exponential fit parameters (inset). 

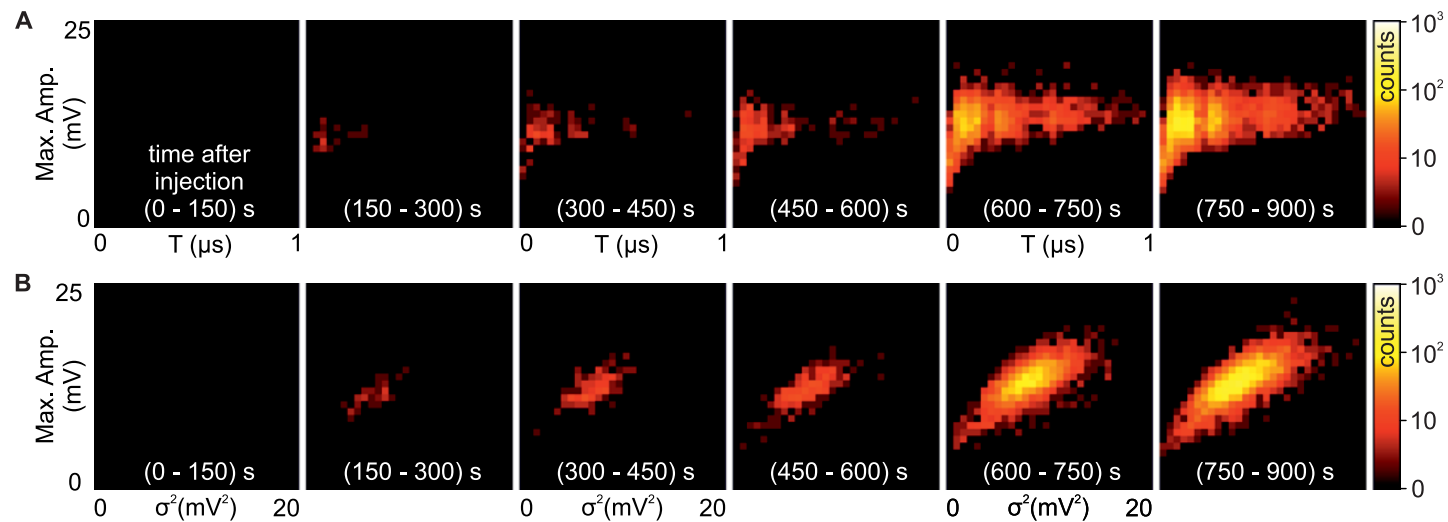

Figure 5. Single gold nanoparticle detection at varying ionic strength: Both panels represent the change of single-particle event properties over time upon injection of sodium chloride (final concentration $30 \mathrm{mM}$ ). (A) Distribution of maximum amplitudes vs event duration. (B) Distribution of maximum amplitudes vs variance $\left(\sigma^{2}\right)$. Based on the same data set as Figure 4 . Scales are linear.

surface. The higher amplitudes contribute stronger to the autocorrelation and therefore give rise to a net decrease in the observed decay time $\tau_{\mathrm{D}}$. This interpretation is further supported by the observed decrease of the stretch exponent $\beta$ with increasing ionic strength, which implies that more and more single-exponential decays with different decay times contribute to the stretched exponential. As mentioned above discrimination between contributions from perturbation rate and perturbation magnitude is not directly possible via autocorrelation contrast alone. Our sensor's capacity to resolve perturbations by single particles, however, provides not only direct access to event rates and amplitudes but also allows us to correlate statistical moments (Figure 5). We find that as the ionic strength increases with time first the number of events increases (Figure $5 \leq 600 \mathrm{~s}$ ), whereas the durations of these events are short and their maximum amplitudes and variances are overall low. This indicates that the analyte particles are at first only gaining access to growing zones of relatively low field strength and thus weak field gradients namely the outer layers of the NR's near field. Starting $600 \mathrm{~s}$ after the injection, the maximum amplitudes and especially the durations of the single-particle perturbations increase significantly toward the end of the measurement (Figure 5A). This shows that the analyte particles can now penetrate significantly deeper into the NR's near field and access zones with higher field strength. The clear correlation between maximum amplitudes and the variance (see Figure 5B) of individual events, as found previously (compare Figure 3E), further supports this interpretation. We think our finding that event duration and amplitude start to increase at higher ionic strength is related to the correlation of local surface charge and local near-field strength. Both are highest on the NR's tips. In consequence, access to the NR's tips might require a higher local ionic strength than access to the sides of the NR and thus occurs later in our measurement. Nonetheless the overall low maximum amplitudes, durations and the absence of the additional sharp tail in the maximum amplitude $v s$ variance distribution as found at higher ionic strength (compare Figure 3E) suggest that the observed NR's tips were not fully accessible at an ionic strength of $30 \mathrm{nM}$. This type of study again shows that our sensor is capable of extracting information beyond the level commonly accessible via ensemble-based measurements and moreover demonstrates our sensor's potential for the future discrimination of analytes with varying charge, for example, via the application of controlled electrostatic potentials throughout the sample cell.

Discussion of Signal to Noise for Single-Protein Detection. In our measurements with a bandwidth of 250 $\mathrm{MHz}$ on single $5 \mathrm{~nm}$ diameter GNPs we typically find signalto-noise ratios (SNR) between 7 and 11 for clear spikes and maximum values reaching up to 16 . For the following estimates we assume a conservative SNR of 9 for these GNPs. The GNPs are 18 times more polarizable (wavelength: $730 \mathrm{~nm}$ ) than a protein of the same dimensions i.e. with a molecular mass of $\sim 60 \mathrm{kDa}$. From this we can estimate an SNR of 1 for a $120 \mathrm{kDa}$ protein at the same bandwidth. This yields a bandwidth-dependent mass detection limit of $7.6 \frac{\mathrm{Da}}{\sqrt{\mathrm{Hz}}}$, i.e., an SNR of 1 for a $76 \mathrm{kDa}$ protein with $10 \mathrm{~ns}$ integration time. In our proof-of-principle measurements we have used a crosspolarized configuration which is not optimal and has a tuning function value of $T \approx 0.7$ (see Supporting Information, section $\mathrm{S} 1$ ). From this, we project that fine-tuning of polarization can yield further improvement of above SNR by a factor $\sim 3$. Moreover if analyte denaturation is not a concern higher powers $>0.02 \mathrm{~mW}$ may be used to further boost the SNR, as long as NR reshaping can be avoided. Taking these considerations into account we estimate a mass detection limit below $2 \frac{\mathrm{Da}}{\sqrt{\mathrm{Hz}}}$, i.e., an $\mathrm{SNR}>1$ for $20 \mathrm{kDa}$ proteins at an integration time of $10 \mathrm{~ns}$.

\section{CONCLUSION}

We have demonstrated the detection of sub-10 nm particles as they undergo Brownian motion through the nanometer-sized near field of single gold nanorods with submicrosecond time resolution. Our sensor is capable to perform ensemble-type measurements in concentration ranges up to $1 \mathrm{mM}$, which are not accessible by label-free image-based techniques. The successful detection of microemulsion nanodroplets with dimensions and optical properties similar to those of $\sim 250$ $\mathrm{kDa}$ proteins shows the promise of our technique for the labelfree detection and identification of biological analytes without specific chemical receptors. The clear bursts obtained from single gold particles further suggest that single-protein molecule recognition will be possible with further optimization. We further found that individual GNRs can exhibit different correlation functions for the same analyte solution. We think this reflects the variation in near-field distributions of 
individual gold nanorods and shows the promise of our method for their characterization. Despite these differences, individual nanorods, once calibrated with a standard or an appropriate optical technique, such as enhanced fluorescence, ${ }^{36}$ may be used for the accurate sizing of analytes. Furthermore, we have demonstrated that our system is capable of recognizing single analyte particles with $5 \mathrm{~nm}$ diameter at lower concentration levels of $\sim 10 \mathrm{nM}$. We have shown that this single-particle resolution provides access to additional layers of information, which are not directly accessible via ensemble methods and hold promise for the future discrimination of particle properties possibly even on a single-shot basis. In principle, our technique does not require the immobilization of the gold nanorods onto a glass surface. Any environment restricting rotational diffusion, e.g., a cell membrane or other fixed structures, could be used to optimize the scattering signal of the nanorod.

\section{METHODS}

Setup. Here we list the components depicted in Figure 1A:

Objective: Olympus UPLFLN100XOP

Tube lens: Olympus super wide tube lens unit

Laser: Coherent 890

APD: A-Cube S500-240 (Laser Components GmbH)

Polarizer LPVISC100 (Thorlabs)

10:90 Beamsplitter BSN11 (Thorlabs)

Glan-Thompson Polarizer GTH10M-B (Thorlabs)

Piezo Translator P-561.3CD (Phyisk Instrumente $\mathrm{GmbH} \&$ Co KG)

Traces were digitized with an oscilloscope (WaveSurfer 24MXs-B, Teledyne Lecroy) and streamed to a PC. Traces with a length of 0.1 ms were typically recorded with at a rate of $5 \times 10^{8}$ samples per second. Consecutive traces were obtained at a rate of 20 traces per second.

Slide Preparation. CTAB-capped gold nanorods were purchased from Nanopartz. GNR stock solutions containing $10 \mathrm{mM} \mathrm{CTAB}$ were sonicated (10 min/Branson 2510) and then deposited onto glass slides (Borosilicate glass diameter $25 \mathrm{~mm}$ thickness No.1, VWR) via spin-coating (Specialty coating Systems Spin Coater 6700). The CTAB-layer was consequently removed via UV-cleaning $(15 \mathrm{~min}$, Jelight Company, Inc. UVO-Cleaner) and the slide was rinsed with Milli-Q water.

Preparation of Gold Nanoparticles. Citrate-capped $5 \mathrm{~nm}$ diameter GNPs were purchased from Nanopartz and sonicated for 10 min before injection.

Preparation of the Microemulsion. The preparation of the soybean oil/polyoxyethylene-10-oleyl ether (Brij-O10)/ water emulsion system was performed in accordance with the work of Warisnoicharoen et al., ${ }^{29}$ i.e., by heating the mixture to $(343-353) \mathrm{K}$ for $10 \mathrm{~min}$ and consequently cooling it down to $298 \mathrm{~K}$, all while continuously stirring the solution. All chemicals were purchased from Sigma-Aldrich. Microemulsions were stored at room temperature.

\section{ASSOCIATED CONTENT}

\section{SI Supporting Information}

The Supporting Information is available free of charge at https://pubs.acs.org/doi/10.1021/acsnano.0c07335.

Description of tuning function, estimate of NR heating, variation of stretched exponentials between NRs, computation of autocorrelations, discussion of autocorrelation contrast, nanodroplet size distributions, description of Debye length, single-event detection and analysis algorithms, distribution of interevent durations, 3Ddiffusion simulation and results, HAADF-STEM images of NRs, NR size distributions, NR scattering spectra (PDF)

\section{AUTHOR INFORMATION}

\section{Corresponding Author}

Michel Orrit - Huygens-Kamerlingh Onnes Laboratory, Leiden University, 2300 RA Leiden, The Netherlands; 이이.org/ 0000-0002-3607-3426; Email: orrit@physics.leidenuniv.nl

\section{Authors}

Martin Dieter Baaske - Huygens-Kamerlingh Onnes Laboratory, Leiden University, 2300 RA Leiden, The Netherlands; 이이이.org/0000-0003-2384-7557

Peter Sebastian Neu - Huygens-Kamerlingh Onnes Laboratory, Leiden University, 2300 RA Leiden, The Netherlands; (1) orcid.org/0000-0001-9569-6220

Complete contact information is available at: https://pubs.acs.org/10.1021/acsnano.0c07335

\section{Notes}

The authors declare no competing financial interest.

\section{ACKNOWLEDGMENTS}

This work was supported by The Netherlands Organisation for Scientific Research (NWO) and has received funding from the European Union's Horizon 2020 research and innovation programme under the Marie Skłodowska-Curie Grant Agreement no. 792595 (MDB). The authors thank Wiebke Albrecht and Sara Bals from the University of Antwerp for providing HAADF-STEM images of nanorod samples.

\section{REFERENCES}

(1) Piliarik, M.; Sandoghdar, V. Direct Optical Sensing of Single Unlabelled Proteins and Super-Resolution Imaging of their Binding Sites. Nat. Commun. 2014, 5, 4495.

(2) Liebel, M.; Hugall, J. T.; van Hulst, N. F. Ultrasensitive LabelFree Nanosensing and High-Speed Tracking of Single Proteins. Nano Lett. 2017, 17, 1277-1281.

(3) Huang, Y.-F.; Zhuo, G.-Y.; Chou, C.-Y.; Lin, C.-H.; Chang, W.; Hsieh, C.-L. Coherent Brightfield Microscopy Provides the Spatiotemporal Resolution to Study Early Stage Viral Infection in Live Cells. ACS Nano 2017, 11, 2575-2585.

(4) McDonald, M. P.; Gemeinhardt, A.; König, K.; Piliarik, M.; Schaffer, S.; Völkl, S.; Aigner, M.; Mackensen, A.; Sandoghdar, V. Visualizing Single-Cell Secretion Dynamics with Single-Protein Sensitivity. Nano Lett. 2018, 18, 513-519.

(5) Young, G.; Hundt, N.; Cole, D.; Fineberg, A.; Andrecka, J.; Tyler, A.; Olerinyova, A.; Ansari, A.; Marklund, E. G.; Collier, M. P.; Chandler, S. A.; Tkachenko, O.; Allen, J.; Crispin, M.; Billington, N.; Takagi, Y.; Sellers, J. R.; Eichmann, C.; Selenko, P.; Frey, L.; et al. Quantitative Mass Imaging of Single Biological Macromolecules. Science 2018, 360, 423-427.

(6) Zijlstra, P.; Paulo, P. M. R.; Orrit, M. Optical Detection of Single Non-Absorbing Molecules Using the Surface Plasmon Resonance of a Gold Nanorod. Nat. Nanotechnol. 2012, 7, 379-382.

(7) Ament, I.; Prasad, J.; Henkel, A.; Schmachtel, S.; Sönnichsen, C. Single Unlabeled Protein Detection on Individual Plasmonic Nanoparticles. Nano Lett. 2012, 12, 1092-1095.

(8) Rosman, C.; Prasad, J.; Neiser, A.; Henkel, A.; Edgar, J.; Sönnichsen, C. Multiplexed Plasmon Sensor for Rapid Label-Free Analyte Detection. Nano Lett. 2013, 13, 3243-3247.

(9) Beuwer, M. A.; Prins, M. W. J.; Zijlstra, P. Stochastic Protein Interactions Monitored by Hundreds of Single-Molecule Plasmonic Biosensors. Nano Lett. 2015, 15, 3507-3511. 
(10) Ye, W.; Götz, M.; Celiksoy, S.; Tüting, L.; Ratzke, C.; Prasad, J.; Ricken, J.; Wegner, S. V.; Ahijado-Guzmán, R.; Hugel, T.; Sönnichsen, C. Conformational Dynamics of a Single Protein Monitored for $24 \mathrm{~h}$ at Video Rate. Nano Lett. 2018, 18, 6633-6637. (11) Vollmer, F.; Arnold, S.; Keng, D. Single Virus Detection from the Reactive Shift of a Whispering-Gallery Mode. Proc. Natl. Acad. Sci. U. S. A. 2008, 105, 20701-4.

(12) Zhu, J.; Ozdemir, S. K.; Xiao, Y.-F.; Li, L.; He, L.; Chen, D.-R.; Yang, L. On-Chip Single Nanoparticle Detection and Sizing by Mode Splitting in an Ultrahigh-Q Microresonator. Nat. Photonics 2010, 4, 46-49.

(13) He, L.; Özdemir, S. K.; Zhu, J.; Kim, W.; Yang, L. Detecting Single Viruses and Nanoparticles Using Whispering Gallery Microlasers. Nat. Nanotechnol. 2011, 6, 428-432.

(14) Shopova, S. I.; Rajmangal, R.; Holler, S.; Arnold, S. Plasmonic Enhancement of a Whispering-Gallery-Mode Biosensor for Single Nanoparticle Detection. Appl. Phys. Lett. 2011, 98, 243104.

(15) Dantham, V. R.; Holler, S.; Barbre, C.; Keng, D.; Kolchenko, V.; Arnold, S. Label-Free Detection of Single Protein Using a Nanoplasmonic-Photonic Hybrid Microcavity. Nano Lett. 2013, 13, 3347-3351.

(16) Li, B.-B.; Clements, W. R.; Yu, X.-C.; Shi, K.; Gong, Q.; Xiao, Y.-F. Single Nanoparticle Detection Using Split-Mode Microcavity Raman Lasers. Proc. Natl. Acad. Sci. U. S. A. 2014, 111, 14657-14662.

(17) Baaske, M. D.; Foreman, M. R.; Vollmer, F. Single-Molecule Nucleic Acid Interactions Monitored on a Label-Free Microcavity Biosensor Platform. Nat. Nanotechnol. 2014, 9, 933-939.

(18) Kim, E.; Baaske, M. D.; Vollmer, F. In Situ Observation of Single-Molecule Surface Reactions from Low to High Affinities. Adv. Mater. 2016, 28, 9941-9948.

(19) Baaske, M. D.; Vollmer, F. Optical Observation of Single Atomic Ions Interacting with Plasmonic Nanorods in Aqueous Solution. Nat. Photonics 2016, 10, 733-739.

(20) Chen, W.; Özdemir, S. K.; Zhao, G.; Wiersig, J.; Yang, L. Exceptional Points Enhance Sensing in an Optical Microcavity. Nature 2017, 548, 192-196.

(21) Kim, E.; Baaske, M. D.; Schuldes, I.; Wilsch, P. S.; Vollmer, F. Label-Free Optical Detection of Single Enzyme-Reactant Reactions and Associated Conformational Changes. Sci. Adv. 2017, 3, No. e1603044.

(22) Ignatovich, F. V.; Novotny, L. Real-Time and Background-Free Detection of Nanoscale Particles. Phys. Rev. Lett. 2006, 96, 1-4.

(23) Krishnan, M.; Mojarad, N.; Kukura, P.; Sandoghdar, V. Geometry-Induced Electrostatic Trapping of Nanometric Objects in a Fluid. Nature 2010, 467, 692-695.

(24) Faez, S.; Lahini, Y.; Weidlich, S.; Garmann, R. F.; Wondraczek, K.; Zeisberger, M.; Schmidt, M. A.; Orrit, M.; Manoharan, V. N. Fast, Label-Free Tracking of Single Viruses and Weakly Scattering Nanoparticles in a Nanofluidic Optical Fiber. ACS Nano 2015, 9, 12349-12357.

(25) Squires, A. H.; Lavania, A. A.; Dahlberg, P. D.; Moerner, W. E. Interferometric Scattering Enables Fluorescence-Free Electrokinetic Trapping of Single Nanoparticles in Free Solution. Nano Lett. 2019, 19, 4112-4117.

(26) Wulf, V.; Knoch, F.; Speck, T.; Sönnichsen, C. Gold Nanorods as Plasmonic Sensors for Particle Diffusion. J. Phys. Chem. Lett. 2016, 7, 4951-4955.

(27) Jollans, T.; Baaske, M. D.; Orrit, M. Nonfluorescent Optical Probing of Single Molecules and Nanoparticles. J. Phys. Chem. C 2019, 123, 14107-14117.

(28) Warisnoicharoen, W.; Lansley, A. B.; Lawrence, M. J. LightScattering Investigations on Dilute Nonionic Oil-in-Water Microemulsions. AAPS PharmSci 2000, 2, 16-26.

(29) Warisnoicharoen, W.; Lansley, A.; Lawrence, M. Nonionic Oilin-Water Microemulsions: the Effect of Oil Type on Phase Behaviour. Int. J. Pharm. 2000, 198, 7-27.

(30) Malcolmson, C.; Barlow, D. J.; Lawrence, M. J. Light-Scattering Studies of Testosterone Enanthate Containing Soybean Oil/
C18:1E10/Water Oil-in-Water Microemulsions. J. Pharm. Sci. 2002, 91, 2317-2331.

(31) Kops-Werkhoven, M. M.; Fijnaut, H. M. Dynamic Behavior of Silica Dispersions Studied Near the Optical Matching Point. J. Chem. Phys. 1982, 77, 2242-2253.

(32) Benz, F.; Schmidt, M. K.; Dreismann, A.; Chikkaraddy, R.; Zhang, Y.; Demetriadou, A.; Carnegie, C.; Ohadi, H.; de Nijs, B.; Esteban, R.; Aizpurua, J.; Baumberg, J. J. Single-molecule Optomechanics in "Picocavities". Science 2016, 354, 726-729.

(33) Vanrompay, H.; Bladt, E.; Albrecht, W.; Béché, A.; Zakhozheva, M.; Sánchez-Iglesias, A.; Liz-Marzán, L. M.; Bals, S. 3D Characterization of Heat-induced Morphological Changes of Au Nanostars by Fast in Situ Electron Tomography. Nanoscale 2018, 10, 2279222801.

(34) Skorikov, A.; Albrecht, W.; Bladt, E.; Xie, X.; van der Hoeven, J. E. S.; van Blaaderen, A.; Van Aert, S.; Bals, S. Quantitative 3D Characterization of Elemental Diffusion Dynamics in Individual Ag@ Au Nanoparticles with Different Shapes. ACS Nano 2019, 13, 1342113429.

(35) Israelachvili, J. N. Intermolecular and Surface Forces; Academic Press: San Diego, 2011.

(36) Caldarola, M.; Pradhan, B.; Orrit, M. Quantifying Fluorescence Enhancement for Slowly Diffusing Single Molecules in Plasmonic near Fields. J. Chem. Phys. 2018, 148, 123334. 